\title{
Interaction Design Teaching Method Design
}

\author{
Chen-Wei Chiang and Kiyoshi Tomimatsu \\ Kyushu University \\ 4-9-1 Shiobaru Minami-ku Fukuoka 815-0032, Japan \\ interaction.tw@gmail.com, tomimatu@design.kyushu-u.ac.jp
}

\begin{abstract}
Recently, Interaction Design became popular. We found that, if we want to improve interaction design, an interaction design education method is indispensible. Especially, interaction design usually consists of two or more different fields of discipline. When members with different backgrounds are working together, there are always cause many problems, mainly during brainstorming processes. In the final, we tried to find out the main cause of the problem and tried to figure out a method for interaction design education. In the end, we also propose the possible solution to improve interaction design teaching design. We except this research can help to improve interaction design education.
\end{abstract}

Keywords: Interaction Design, workshop.

\section{Introduction}

Based on past records of conducting interaction design workshops, we identify three main problems that are commonly encountered by the group members, i.e.: (1) Different professional backgrounds; each member needed to spend more time to understand each other's profession. (2) Members were inefficient in developing ideas and brainstorming. (3) The results of the workshops were not as expected because of repeated discussions and readjusted ideas. Students have made comments that they would like to learn new technologies or new design methods, but due to lack of time, discussions among members had to be shortened and created unexpected results.

This research will analyze the problems that group members encounter during the interaction workshop brainstorming, such as spending most of their time developing and discussing ideas. This research is to create a teaching method to help students achieve better results and be more efficient at brainstorming.

\section{Background}

\subsection{Related Work}

There are several of researches, projects, and courses related to Interaction Design teaching method which are aimed to the students without former knowledge of Computer Science. 
In an education-friendly construction platform for wearable computing project, Ngai et al. [1] presented a programmable platform TeeBoard for wearable computing. This TeeBoard is used for training students to integrate their knowledge with physical computing.

Miyata et al.[2] have designed an education method for VR content creation for students. In this course, the education is aimed to students with the computer science background. They implied that cooperation is an indispensible element in designing contents in workshop. Concurrent thinking allows students to share their ideas through group discussion.

Ariga et al.[3] designed the course for students who are majoring in art. A toolkit was designed for students in this course which consists of both the software and hardware. They attempted to introduce interaction design to art students by using this toolkit. They designed two different versions of toolkit for their course. The second version is an improved version of the first one. Using this version, it was much easier to use sensors for students to create original interaction arts and designs.

In this course, Ariga et al. divided the toolkit's hardware into four separate elements, i.e.: I/O modules, Inputs, Outputs, and Wires. Compared to the first version, the second version of the toolkit reduced some electronic parts of Input and Output elements. Furthermore, they mentioned that the simplification setting could also aid students to understand the input and output processes.

Embodied and tangible interaction design teaching method has been discussed by Klemmer et. al.[4] and Kenneth[5]. Klemmer et. al. developed a Human-Computer Interaction Design Studio course, which is aimed to undergraduates students with HCI background, but with limited exposure to practice. The course was structured in four projects and each project is aimed to teach different aspect of embodied computing. By applying this diverse approach, the students will be able to incorporate their understanding and develop their interaction designing skills further.

Furthermore, by the classification and taxonomy of embodied and tangible interfaces was discussed by Kenneth, providing a clear guideline for the taxonomy of tangible interfaces. By applying metaphors to embodied interfaces, embodied interfaces can be plotted visually for different tasks. This taxonomy would acts as a useful tool for educators to organize their concepts before put them into real practices.

\subsection{Previous Research}

In Computational Thinking[6], Jeannette Wing implied that computer science education could be applied to different fields. Furthermore, Guzdial's[7] mentioned that educators need to make Computational Thinking (CT) to be accessible for everyone. Further considerations should be taken when we consider about how to introduce Interaction Design to students without computer science background. Furthermore, we also used the key questions identified by Guzdial as the considerations for our course design, i.e.:

1. What do non-computing students understand about computing?

2. What kind of challenges will they find?

3. What kinds of tools can make them access CT easily?

4. How should we, as educators organize and structure our courses to make computing accessible to the broad range of students? 
Recent report in 2009[8] by Computer Science \& Engineering (CISE) division of the National Science Foundation (NSF) mentioned the importance of CT. In fact, CT has been widely acknowledged not only in the field of Computer Science but also in other disciplines. The author proposed a CT based creative multi-media design educational course. The basic knowledge in multi-media education includes the knowledge of graphic, sound, and video. In this course designing, a new educating process is proposed in multi-media education, which includes technical skills, physical computing, and concept generation.

In this paper, we emphasize on physical computing education, i.e.: teaching the students on how to apply physical computing on their work. As for the final output for this education method, students will be able to integrate physical computing techniques with their design.

\section{The definition of workshop}

In general, workshops are teaching methods that divide students in groups for a short period of time. The interaction design teaching method can help students with different backgrounds develop new concepts in group discussions to create final projects. In this research we define an interaction workshop as a workshop that meets these three conditions below:

1. Continuous four days workshop.

2. Students must have computer science and media design background.

3 . Every group must have a final interaction design project finished upon completion of the workshop.

\section{The Methods of Past Interaction Design Workshops}

From analyzing the information, we can conclude some important aspects of interaction design workshops and design the basic syllabus. This is to see if the syllabus can improve students at the first stage of the workshop, so they can be more efficient at developing concepts. From the documents of past workshops, we can conclude the aspects which are described below:

1. Subject: We would use subjects from one or two different areas to integrate into interaction design.

2. Analyzing students' backgrounds: Students background and their professions were analyzed, we divided them into groups so that they can exchange their information to the members that have different backgrounds.

3. Projects presentation: The end results of the workshops were functional prototypes. They were interaction design products or interaction science art installations.

Based on these aspects, we will design the syllabus and set up the basic rules to the students in our experiments. 


\section{Workshop Course}

\subsection{Workshop-1: Media Design and Physical Computing}

\section{The Introduction to Workshop}

In this phase, we introduce basic physical computing tools to the workshop members. The goal is to combines physical computing technology with their professional competence, in addition to improving their interaction design skills.

\section{Workshop Equipment Setting}

In our course, we decided to use Arduino as our physical computing tool. Arduino is an open source physical computing tool, thus we could access many source code and information about Arduino and applied it to the workshop with ease. The participants were not accustomed to physical computing because they are majoring in multi-media design.

\section{Tools and Equipment}

The tools that we used in this course were consists of a hardware and a software. We used arduino as the hardware and Max/Msp as the software.

Arduino is a well-known open source tool for physical computing which means there are many references on internet. There are also many sensor modules that can be easily found on the internet. Otherwise, the students can develop it themselves by studying these references. Students can also learn it effectively in a short time, and apply those what they have learned into their works.

Max/Msp is the graphical program software where people can edit program by moving and combing some blocks than make programs come true. For beginners, this software is good for understanding how the program working. After understand how the program working, they can make their ideas come true by using this tool easily. Let their concepts or prototypes to be much more completely.

\section{Prototyping}

We divided the participants into groups and each group consists of 4-5 members. We also decided the final expected outcome for the course. In this course, our members have to think about their original concepts and finally they should make a prototype based on their original concept. The participants were expected to finish the prototype at the end of the course.

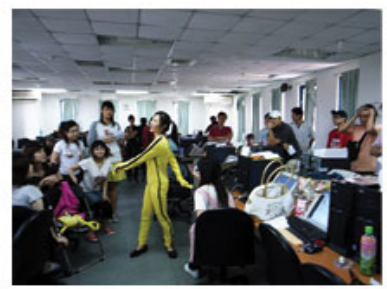

(a)

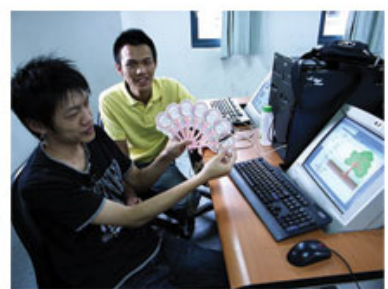

(b)

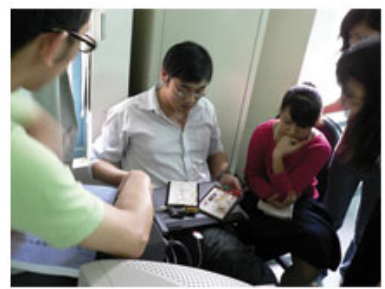

(c)

Fig. 1. (a) Wearable clothing combine with physical computing. (b) Animation controller combine with physical computing. (c) Arduino group studying. 


\section{Conclusion}

Considering that the students were inexperienced in physical computing, teaching them to create a working prototype is more important than developing physical computing skills.

As the final outcome, most of the participants were able to make a working prototype based on their initial ideas. We conclude that they were able combine their concepts successfully with physical computing. However, we found that our members were giving up good concepts during the brainstorming. They thought that because of their limited physical computing and program design they couldn't realize their concepts into working prototypes.

\subsection{Workshop-2: Choreography and Physical Computing}

\section{The Introduction to Workshop}

In this course, we introduce Choreography of interaction to our workshop members. This concept is from Sietske Klooster et al. [9] as they designed movements and introduced them to the design of interaction, developing both as the integrated parts. In our course, we tried to let our members can understand the elements of dance and apply these element into their works for improving their interaction concepts. These concepts must come from the elements of dance and body movements.

\section{Workshop Setting}

According to Sietske Klooster et al. [9], a professional dancer is required to introduce the basic elements of dance for our workshop members. From the dance course, we identified four main elements, i.e.: Body, Space, Time, and Effort. By utilizing these elements, we had our workshop members to consider their interaction works concepts.

\section{Equipment and Tools}

Similar to the first workshop, we still used Arduino and some simple switch for the participants as the main hardware. We assumed that we could be able to reduce the gap of physical computing between the participants who have no physical computing background or experiences in the past.

\section{Prototyping}

We divided the participants into groups and each group consists of 4-5 members. We also decided the final expected outcome for the course. In this course, our members have to think about their original concepts and finally they should make a prototype based on their original concept. The participants were expected to finish the prototype at the end of the course.

\section{Conclusion}

In this workshop, we focused on how well the participants apply the dancing elements to their interaction works. As the evaluation criteria, all of the four elements should be applied on their final works. At the final presentation, the participants could submit several original works which incorporates dancing elements into interaction design. 


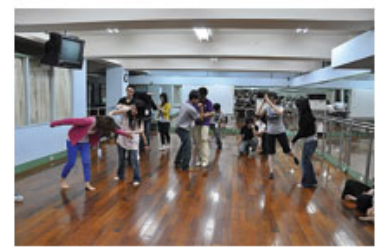

(a)

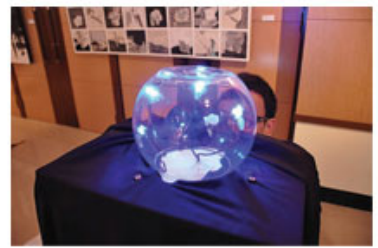

(b)

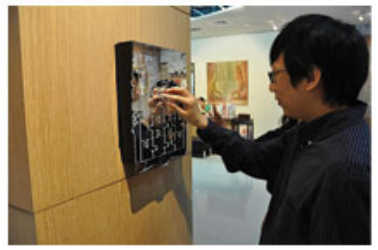

(c)

Fig. 2. (a) Dance course. (b) Work consider about space. (c) Work consider about effort.

\subsection{Workshop-3: Resonance and Computing}

\section{The Introduction to Workshop}

The theme of this workshop is about resonating elements. We attempted to have the participants observing the structure of resonation, applying their resonation experiences into interaction design works. In this workshop, the main objective is resonating and we defined three sub-objectives, i.e.: feeling, thinking and activating.

\section{Workshop Setting:}

In this section, the details of three sub-objective targets, i.e.: feeling, thinking and activating will be discussed in detail:

1. Feeling: using physical computing to represent peoples feeling.

2. Thinking: using wearable clothing to realize interaction design concepts. We consider wearable clothing is like human skin, thus we can represent humans feeling by using wearable clothing.

3. Activating: social network is not only exists in human society, it also exists in resonating. We tried to introduce these communicating behaviors for our members. Trying to discover what we haven't noticed sociality behavior in resonating.

In this course, we tried to let our workshop members compare resonating elements with technological elements. After the comparison, we tried to let our members to simulate the resonating elements by using technological elements and applying these elements into their interaction works.

\section{Equipment and Tools}

We used physical computing to simulate resonating environment. We used wearable clothing to represent humans feeling. As for the activating elements, we tried to have the participants simulate society behaviors.

\section{Prototyping}

We divided the participants into groups and each group consists of 4-5 members. We also decided the final expected outcome for the course in this course, our members have to think about their original concepts and finally they should make a prototype based on their original concept. The participants were expected to finish the prototype at the end of the course. 


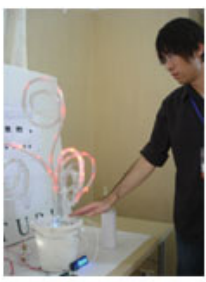

(a)

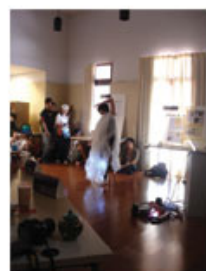

(b)

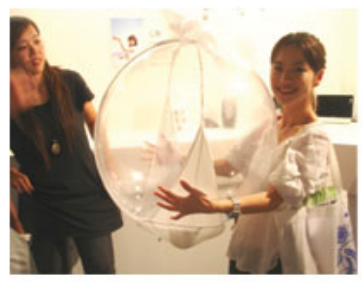

(c)

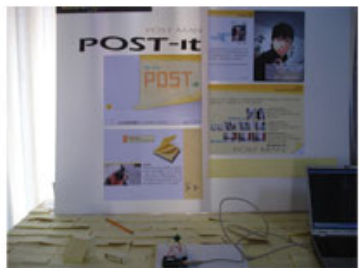

(d)

Fig. 3. (a) Traffic monitor tree. (b) Dance movement with clothing. (c) Body movement with ball. (d) Stick a post on someone's back and get response.

\section{Conclusion}

In the final, we let our members to combine resonating elements with technology elements to create interaction works. In this workshop, we can understand, combining different fields into interaction design could create and help us to consider about that we haven't think about.

\section{Lessons Learned}

\subsection{The Method of Past Interaction Design Workshops}

During the concept development and brainstorming processes, we wrote down our ideas and discussions. In the discussion process, the main subjects changed drastically and diverge the main conversation into unrelated subjects. Sometimes group members would use post-it notes to write down ideas, but it still had the chance of making the main discussion unclear. Members would spend more time on discussions and readjust their ideas and might have to redo the concept developing process. These made the workshops inefficient and subjects could not move to the next level of the syllabus. This research will focus on improving discussion efficiency of effective group discussions.

\subsection{Consider a Method for Improving Interaction Design Workshops}

The training hours in a workshop usually are not long. Therefore, if we want to make workshop to be more effective, improving discussion effectiveness can be a helpful method for reducing time waste and let members have enough time for working and improve the quality of final presentation.

\section{Education Method Design for Workshop}

\subsection{Education Method Design}

Because interaction design involves various areas of knowledge, we will need to use some special terms that are not easy to understand. As the discussion progresses, it 
might become aimless and lower both the discussion efficiency and learning effectiveness. We propose the following methods to improve the process of the interaction workshops so that students can discuss more efficiently, reach the goals quickly, and have better final projects. The methods are:

1. Select keywords to analyze goal elements. For example, if the goal is to make an interaction design in a classroom environment, students would need to describe the space in words and record that information, such as: blackboards, chalks, chairs, desks, etc.

2. Students should record the possible ways of interacting with those objects, such as: touch, press, push, etc.

3. Analyze what computer technologies are suitable for the project and record that information, such as: light sensor, pressure sensor, temperature sensor, etc.

4. Gather the term, randomly pick one from each group, combine the words, and discuss the feasibility, such as how chairs, push, and pressure sensor can be combined to affect interaction design.

Following these steps to start discussions will help to shorten discussion time and improve the quality of final projects.

\subsection{Applying to Workshop}

According this interaction design education method, we applied this method to a group work concept brainstorming. In the final, we got response from workshop members. The response as the following:

1. This method for brainstorming is a very interesting method. It can help us to think about something we never think about. But, if we can have more information about these keywords, it could be more helpful for us.

2. This is an interesting thinking method for making concepts. It let me to think about what I never think about content, and I think this thinking method can help me to improve my creativity.

3. I always think my thinking method is very hard to change, but after I learned this method, I can think something I have never think very easily. I think this method can help me reach my goal of design concept much more easily. It is a very important method for me.

\section{Conclusion}

The process of discussion requires a lot of recording. In the future, this method can be applied as an iPad application for this research. The iPad can provide mobility to students, create and store databases easily for future references. Furthermore, This application can record the terms, then randomly select and combine terms for group discussions. 


\section{References}

1. Ngai, G., Chan, S.C.F., Cheung, J.C.Y., Lau, W.W.Y.: An Education-Friendly Construction Platform for Wearable Computing. In: CHI EA 2009: Proceedings of the 57th International Conference Extended, Abstracts on Human Factors in Computing Systems, Boston, USA, pp. 3235-3240 (2009)

2. Miyata, K., Umemoto, K., Higuchi, T.: An Education Method for VR Content Creation using Groupwork. In: SIGGRAPH Asia 2009, Yokohama Japan (2009)

3. Ariga, T., Mari, K.: Learning Course for Sensory Interaction. In: SIGGRAPH Asia 2009, Yokohama Japan (2009)

4. Klemmer, S.R., Verplank, B., Ju, W.: Teaching Embodied Interaction Design Practice. Journal of Computing Sciences in Colleges 25 (2010)

5. Fishkin, K.P.: A Taxonomy for and Analysis of Tangible Interfaces. Personal and Ubiquitous Computing 8, 347-358 (2004)

6. Wing, J.M.: Computational Thinking. Communications of The ACM 49(3) (2006)

7. Guzdial, M.: Paving the Way for Computational Thinking. Communications of the ACM 51(8), 25-27 (2008)

8. Dann, W., Cooper, S.: Alice 3: Concrete to Abstract. Communications of the ACM 52(8), 27-29 (2009)

9. Klooster, S., Overbeeke, C.J., Sharp, R.: Designing Products as An Integral Part of Choreography of Interaction 2005: The Product's Form As An Integral Part of Movement. In: Proceedings of Design and Semantics of Form and Movement, 1st International Workshop on Design and Semantics of Form and Movement, Newcastle UK, pp. 23-35 (2005) 